Supporting Information for

\title{
Constructing and Transferring Two-dimensional Tessellation Kagome Lattices via Chemical Reactions on $\mathrm{Cu}(111)$ Surface
}

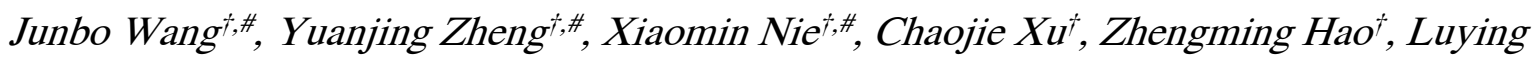
Song ${ }^{\dagger}$, Sifan $\mathrm{You}^{\dagger}$, Jiahao Xit, Minghu Pan ${ }^{\dagger}$, Haiping Lin ${ }^{\dagger,+}$, Youyong L $i^{\dagger}$, Haiming Zhang ${ }^{\dagger}$, Qing Li $^{*}, *+$, Lifeng Chi ${ }^{*}+$

\section{AUTHOR ADDRESS}

†nstitute of Functional Nano \& Soft Materials (FUNSOM), Jiangsu Key Laboratory for Carbon-Based Functional Materials \& Devices, Soochow University, 199 Ren'ai Road, Suzhou, 215123 P. R. China

${ }^{\ddagger}$ School of Physics and Information Technology, Shaanxi Normal University, Xi’an 710119, China

\section{AUTHOR INFORMATION}

\section{Corresponding Author}

*Qing Li: liqing@ snnu.edu.cn

*Lifeng Chi: chilf@ suda.edu.cn 


\section{Methods}

STM measurements. All the experiments were performed with commercial low temperature scanning tunneling microscopy (Unisoku LT-STM 1500s) at a base pressure $1 \times 10^{-10}$ Torr. The $\mathrm{Cu}(111)$ surface was cleaned by cycles of sputtering with $\mathrm{Ar}^{+}$ions and subsequent annealing to $730 \mathrm{~K}$. The tungsten STM tip was cleaned by e-beam heating under UHV. The ABPCA molecules were purchased from J \& K Company (purity higher than 95\%) and were deposited on the surfaces with an organic evaporator. All STM images were acquired at $77 \mathrm{~K}$ and analyzed by WSxM software. ${ }^{1}$ All the $d I / d V$ measurements were conducted with a lock-in amplifier with a voltage modulation of $20 \mathrm{mV}$ and frequency $731 \mathrm{~Hz}$.

Theoretical calculations. Our first-principles calculations were carried out by Vienna ab initio Simulation Package (VASP) code within the framework of density functional theory (DFT). ${ }^{2-}$

${ }^{3}$ To describe the exchange and correlation functional, the generalized gradient approximation (GGA) in the Perdew-Burke-Ernzerhof (PBE) form was employed. ${ }^{4}$ The DFT-D3 functional was used to evaluate the van der Waals (vdW) interactions. ${ }^{5}$ The $\mathrm{Cu}(111)$ periodic slab was consisted of three atomic layers and a vacuum of $15 \AA$. The plane wave energy cutoff was set to $450 \mathrm{eV}$, and the $\Gamma$ point of the Monkhorst-Pack scheme is employed to sample the reciprocal space in the Brillouin zone. The convergence of energy and force were set to $1 \times 10^{-4} \mathrm{eV}$ and $0.02 \mathrm{eV} / \AA$, respectively. 


\section{1, Large-scale STM image of $\alpha$ phase}

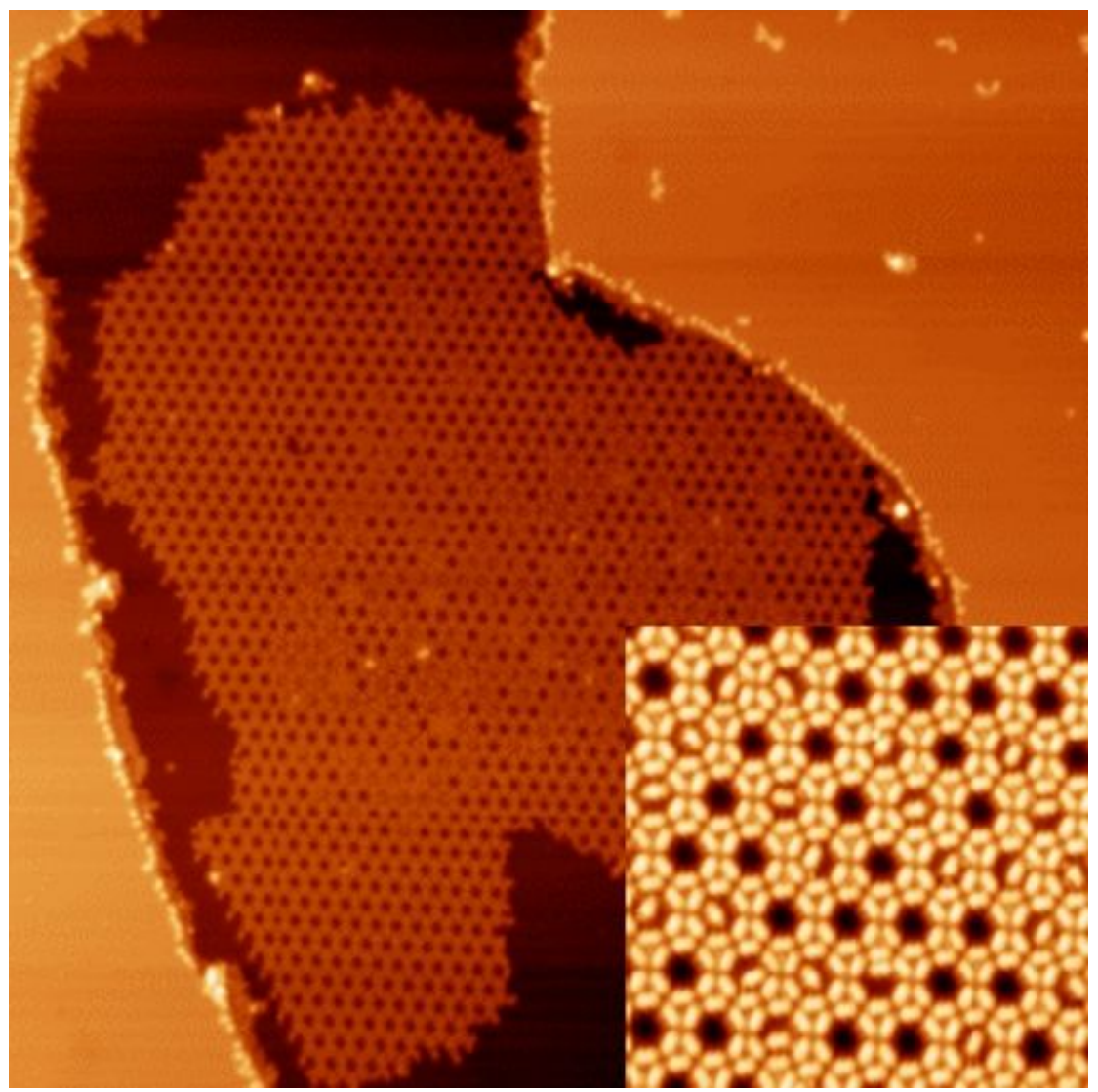

Figure S1. Large-scale STM image of $\alpha$ phase. Insert: zoomed-in STM image of $\alpha$ phase. Tunneling parameters are $I_{\mathrm{t}}=20 \mathrm{pA}, V_{\mathrm{b}}=-1.0 \mathrm{~V}$. The image sizes are $150 \times 150 \mathrm{~nm}^{2}$ for the main image and $20 \times 20 \mathrm{~nm}^{2}$ for its inset. 


\section{2, Large-scale STM image of $\beta$ phase}

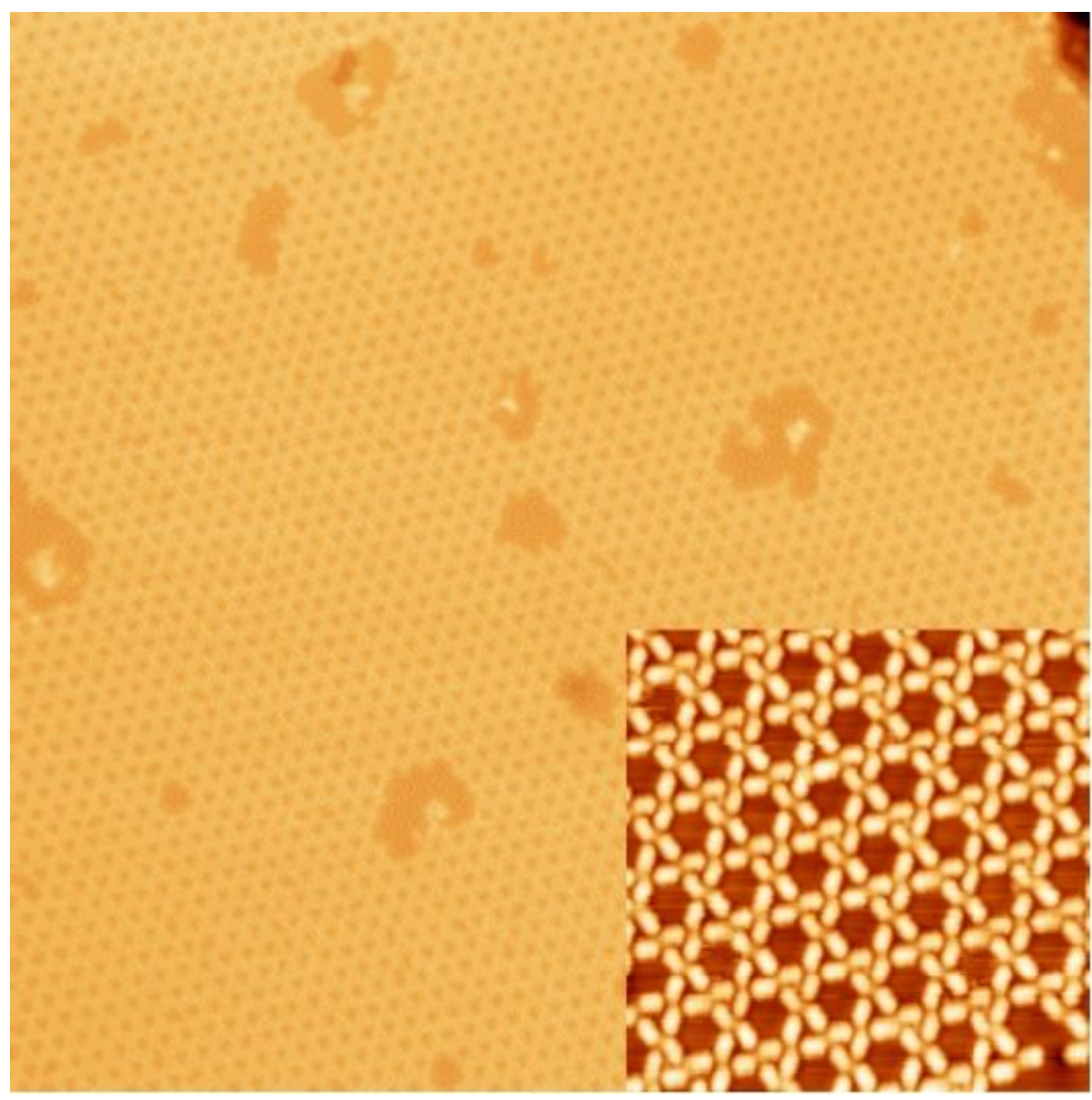

Figure S2. Large-scale STM image of $\beta$ phase. Insert: zoomed-in STM image of $\beta$ phase. Tunneling parameters are $I_{\mathrm{t}}=20 \mathrm{pA}, V_{\mathrm{b}}=-1.0 \mathrm{~V}$. The image sizes are $150 \times 150 \mathrm{~nm}^{2}$ for the main image and $20 \times 20 \mathrm{~nm}^{2}$ for its inset. 


\section{3, Evidence of the formation of $\mathrm{N}-\mathrm{Cu}-\mathrm{N}$ bonds}

Figure S3 gives the STM (Fig. S3a) and AFM (Fig. S3b) images of the N-Cu-N bonds on a $\mathrm{Cu}(111)$ surface. The $\mathrm{Cu}$ ad-atom exhibits as dark dot in the STM image, as highlighted by the arrow in Fig. S3a. The formation of the $\mathrm{N}-\mathrm{Cu}-\mathrm{N}$ bonds are further confirmed by the AFM image shown in Fig. S3b, and STM image simulations and XPS results in our previous report. ${ }^{6}$

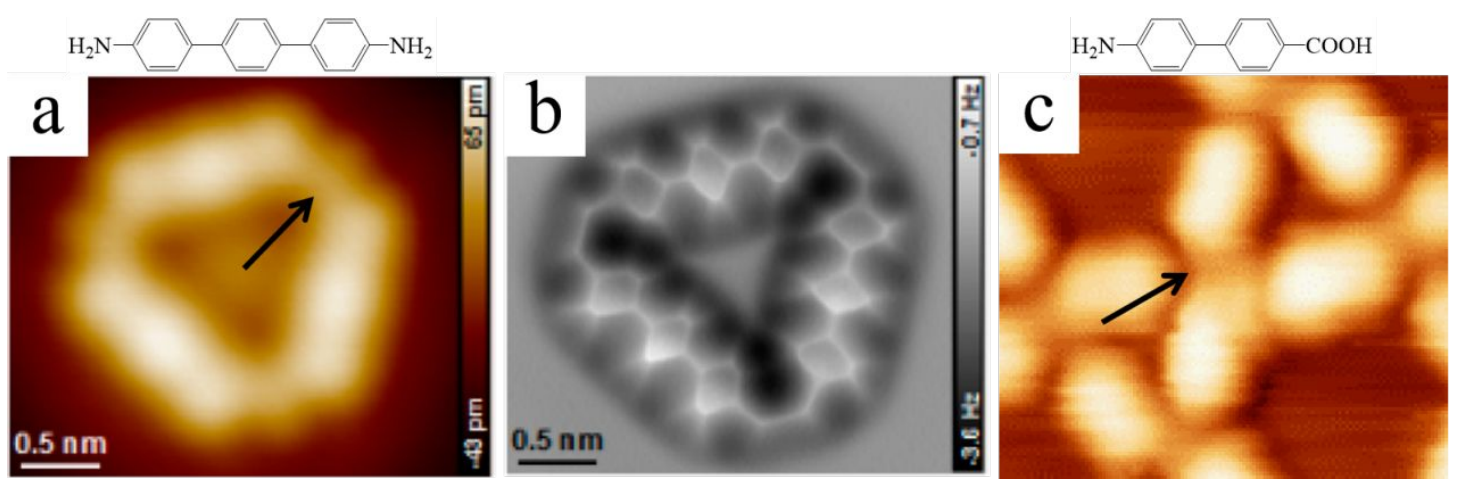

Figure S3. (a) STM topographic image of the trimer structure formed by three DATP monomers on a copper surface. (b) the corresponding nc-AFM frequency shift image of (a). (c) Highly resolved STM image of the ABPCA dimers. The image size is $3.5 \times 3.2 \mathrm{~nm}^{2}$ for (c). 
4, Surface state of the ABPCA adsorbed Cu(111) surface

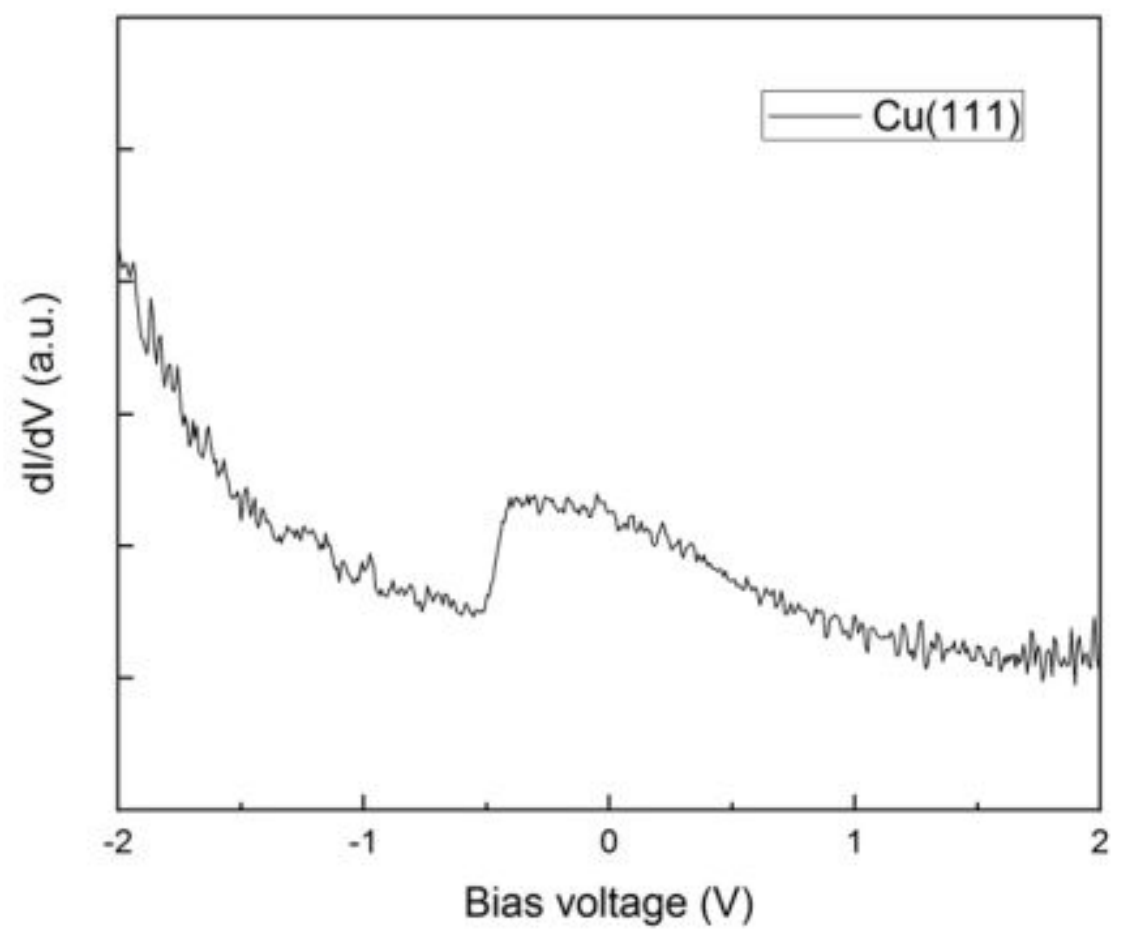

Figure S4. The $d I / d V$ curve acquired on a ABPCA adsorbed $\mathrm{Cu}(111)$ surface. The modulation voltage is $V_{\mathrm{rms}}=20 \mathrm{mV}$ ). 


\section{$5, d I / d V$ images $\alpha$ phase}

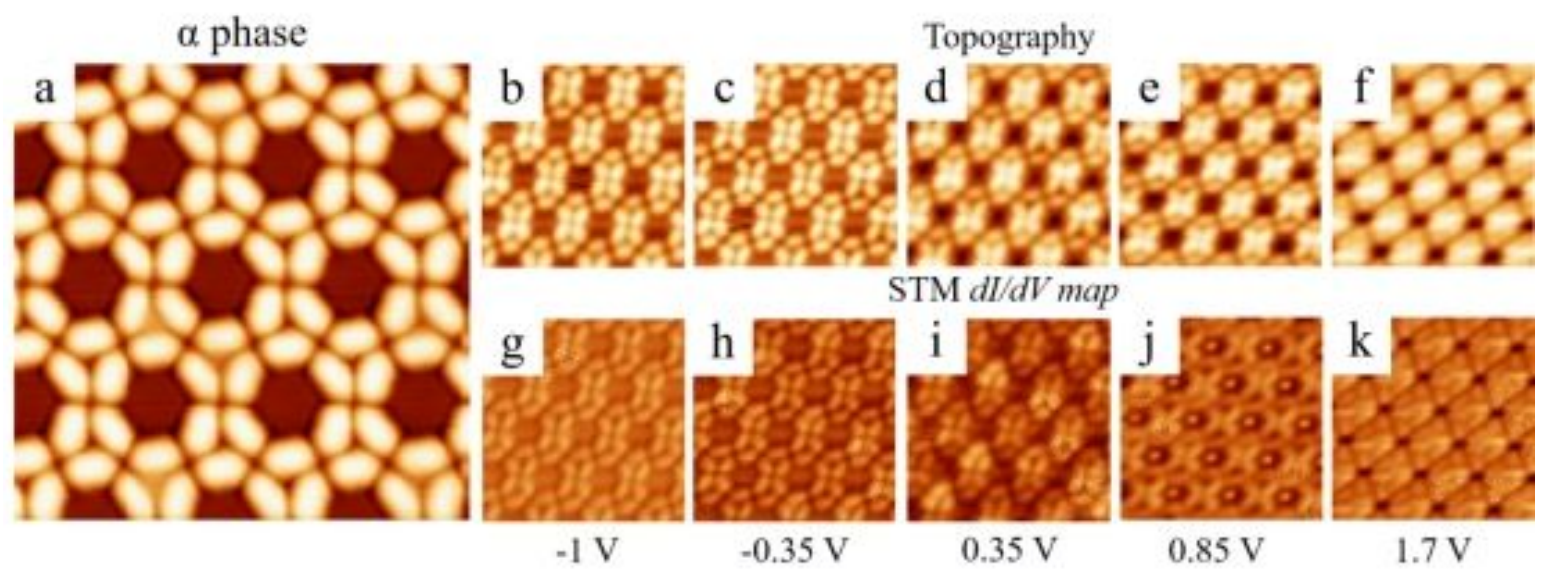

Figure S5. (a) High- resolution STM image of $\alpha$ phase obtained at -1 V. (b)-(f) STM topographic image taken at $-1 \mathrm{~V},-0.35 \mathrm{~V}, 0.35 \mathrm{~V}, 0.85 \mathrm{~V}$ and $1.7 \mathrm{~V}$, respectively. (g)-(k) The corresponding $d I / d V$ images of (b)-(f). The sizes are $10 \times 10 \mathrm{~nm}^{2}$ for all the images. The modulation voltage is $V_{\mathrm{rms}}=20 \mathrm{mV}$ ). 


\section{$6, d I / d V$ images $\beta$ phase}
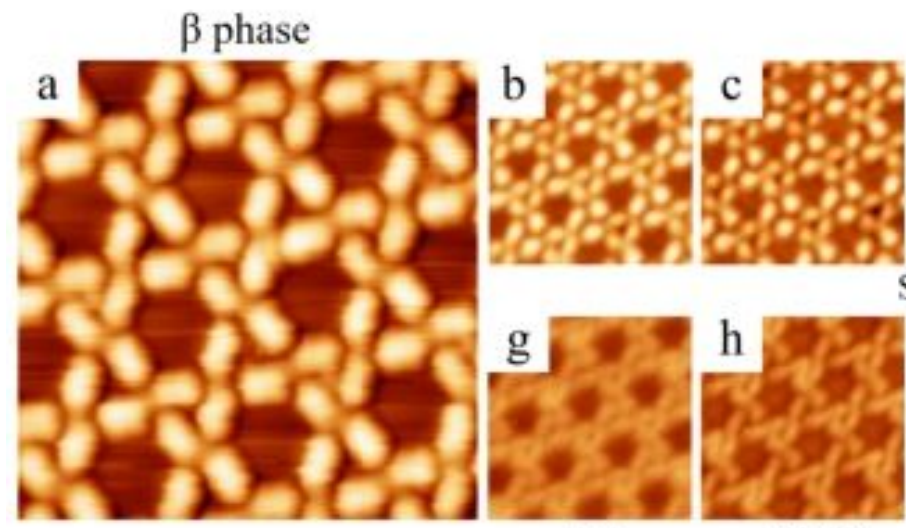

Topography

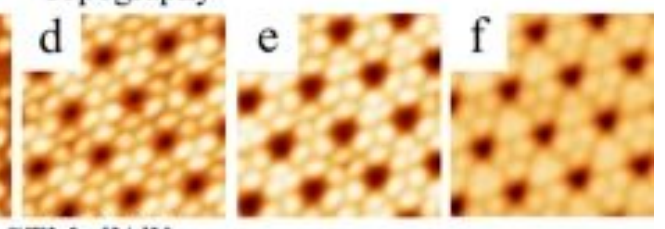

$\mathrm{STM} d l / d V$ map

$-1 \mathrm{~V}$

$-0.35 \mathrm{~V}$

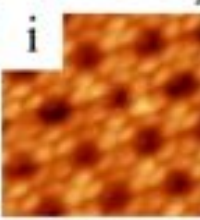

$0.35 \mathrm{~V}$

$0.85 \mathrm{~V}$

$1.7 \mathrm{~V}$

Figure S6. (a) High- resolution STM image of $\beta$ phase obtained at -1 V. (b)-(f) STM topographic image taken at $-1 \mathrm{~V},-0.35 \mathrm{~V}, 0.35 \mathrm{~V}, 0.85 \mathrm{~V}$ and $1.7 \mathrm{~V}$, respectively. (g)-(k) The corresponding $d I / d V$ images of (b)-(f). The sizes are $10 \times 10 \mathrm{~nm}^{2}$ for all the images. The modulation voltage is $V_{\mathrm{rms}}=20 \mathrm{mV}$ ). 


\section{REFERENCES}

(1) Horcas, I.; Fernandez, R.; Gomez-Rodriguez, J. M.; Colchero, J.; Gomez-Herrero, J.; Baro, A. M. WSXM: a software for scanning probe microscopy and a tool for nanotechnology. Rev. Sci. Instrum. 2007, 78, 013705.

(2) Furthmuller, J.; Kresse, G. Efficiency of ab-initio total energy calculations for metals and semiconductors using a plane-wave basis set. Comput. Mater. Sci. 1996, 6, 15-50.

(3) Kresse, G.; Furthmuller, J. Efficient iterative schemes for ab initio total-energy calculations using a plane-wave basis set. Phys. Rev. B 1996, 54, 11169-11186.

(4) Perdew, J. P.; Burke, K.; Ernzerhof, M. Generalized Gradient Approximation Made Simple. Phys. Rev. L 1996, 77, 3865-3868.

(5) Grimme, S.; Antony, J.; Ehrlich, S.; Krieg, H. A consistent and accurate ab initio parametrization of density functional dispersion correction (DFT-D) for the 94 elements H-Pu. J. Chem. Phys. 2010, 132, 154104.

(6) Li, Q.; Yang, B.; Bjork, J.; Zhong, Q.; Ju, H.; Zhang, J.; Cao, N.; Shi, Z.; Zhang, H.; Ebeling, D.; et al. Hierarchical dehydrogenation reactions on a copper surface. J. Am. Chem. Soc. 2018, 140, 6076-6082. 